\title{
Prediction of glucose excursions under uncertain parameters and food intake in intensive insulin therapy for type 1 diabetes mellitus
}

\author{
R. Calm, M. García-Jaramillo, J. Vehí, J. Bondia, C. Tarín and W. García-Gabín
}

\begin{abstract}
Considering the difficulty in the insulin dosage selection and the problem of hyper- and hypoglycaemia episodes in type 1 diabetes, dosage-aid systems appear as tremendously helpful for these patients. A model-based approach to this problem must unavoidably consider uncertainty sources such as the large intra-patient variability and food intake. This work addresses the prediction of glycaemia for a given insulin therapy face to parametric and input uncertainty, by means of modal interval analysis. As result, a band containing all possible glucose excursions suffered by the patient for the given uncertainty is obtained. From it, a safer prediction of possible hyper- and hypoglycaemia episodes can be calculated.
\end{abstract}

\section{INTRODUCTION}

Diabetes mellitus is a metabolic disease that is accompanied by elevated plasma glucose levels comprising all forms of acute or chronic hyperglycaemia. This is so due to the lack of insulin secretion by the $\beta$-cells in the islets of Langerhans in the pancreas (type 1 diabetes) or a reduction in its efficiency to promote transport of glucose into the cells (type 2 diabetes).

Since the Diabetes Control and Complications Trial [1], euglycaemia has been established as the control objective for patients with type 1 diabetes, except if some contraindication exists. However, there still lacks a universal, efficient and safe system able to normalize the glucose levels of patients. The intensive insulin therapy required to achieve the glucose control objectives, based on the injection of basal and bolus insulin to "emulate" its physiological secretion, has as counter-action an increase in the risk of severe hypoglycaemia with all their consequences.

Although several rules do exist for the calculus of the bolus insulin dose, and even these ones have been lately incorporated for automatic calculus in some insulin pumps, trial-and error adjustments of the therapy must be carried out. Considering the difficulty in the insulin dosage selection and the problem of hyper- and hypoglycaemia episodes, dosageaid systems appear as tremendously helpful for patients with type 1 diabetes.

This work has been supported by the Spanish government under the grants DPI-2004-07167-C02-01 and DPI-2004-07167-C02-02, by the catalan government under grant SGR-00296 and by the European Union through FEDER funds

R. Calm, M. García-Jaramillo, J. Vehí and W. García-Gabín are with the Institut d'Informatica i Aplicacions, University of Girona, Girona, Spain remei.calm@udg.es, josep.vehi@udg.es, magarcia@eia.udg.es, wgarcia@eia.udg.es

J. Bondia is with the ai2 Institute, Technical University of Valencia, Valencia, Spain jbondia@isa.upv.es

C. Tarín is with the iTEAM Institute, Technical University of Valencia, Valencia, Spain critasa@iteam.upv.es
Such a system must rely on accurate enough predictions of glycaemia. However, there exists a large intra-individual and inter-individual variability in the patients' behavior. Other important source of uncertainty is the food intake (grams of carbohydrates) since it is difficult to have a precise estimation from a mixed meal.

This makes necessary the development of prediction tools able to consider different sources of uncertainty (input, parameters, initial state). In this work, modal interval analysis has been applied for the plasma glucose prediction face to uncertain food intake and patient parameters such as insulin hepatic and peripheral sensitivity. As result, upper and lower envelopes of all the possible glucose excursions suffered by the patient, as predicted by the model, are obtained. This prediction can be used by patients to evaluate the result of different insulin therapy decisions, and evaluate the risk of hypoglycaemia and hyperglycaemia within the framework of a dosage-aid system.

\section{GLUCORREGULATORY MODEL}

Simulation of an insulin therapy involves modelling subcutaneous insulin absorption, carbohydrates digestion and absorption, insulin pharmacokinetics and pharmacodynamics (PK/PD), and glucose metabolism. The relationship among these processes is shown in Figure 1.

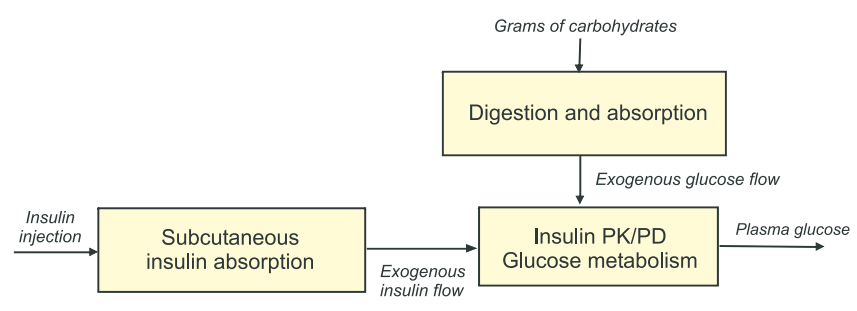

Fig. 1. Model overview.

In this work, the models presented in [8], for subcutaneous insulin absorption, and [5], for the rest of the components of the model, have been combined to represent the glucorregulatory model. In both cases, experimental validation results have been reported in the literature.

Subcutaneous insulin absorption. Given and insulin therapy, i.e. a set of basal and bolus insulin doses and injection times, this model calculates how the injected insulin appears in blood (exogenous insulin flow). The model in [8] has been selected since it comprehends long- and intermediate-acting insulin preparations, used as basal insulin, as well as rapidly- and short-acting (regular) insulin, used as bolus insulin. The reader is referred to [8] for a 
detailed description of this model. As result, the exogenous insulin flow, $I_{\mathrm{ex}}(t)=I_{\mathrm{ex}}^{\mathrm{bolus}}(t)+I_{\mathrm{ex}}^{\mathrm{basal}}(t)$ is obtained.

Carbohydrates digestion and absorption. This model describes the carbohydrates catabolism to monosaccharide (mostly glucose) taking place during meal digestion, as well as intestinal absorption. The model considered here is that described in [5], where the glucose absorption rate $U_{G}$ is represented by

$$
U_{\mathrm{G}}(t)=\frac{D_{G} A_{G} t \exp \left(-t / t_{\max , G}\right)}{t_{\max , G}^{2}}
$$

being $D_{G}$ the amount of carbohydrates ingested, $A_{G}$ is carbohydrate bioavailability and $t_{\max , G}$ is the time-of-maximum appearance of glucose in plasma.

Insulin PK/PD and glucose metabolism. Insulin pharmacokinetics, either basal or bolus, is considered of firstorder. Plasma insulin concentration, $I(t)$, is thus described as

$$
\frac{\mathrm{d} I(t)}{\mathrm{d} t}=\frac{I_{\mathrm{ex}}(t)}{V_{I}}-k_{e} I(t)
$$

where $I_{\mathrm{ex}}(t)$ is the exogenous insulin absorption rate abovedescribed, $k_{e}$ is the fractional elimination rate and $V_{I}$ is the insulin distribution volume.

Plasma insulin concentration is considered to affect on glucose transport from plasma to the tissues, hepatic glucose production and peripheral glucose disposal [5]. These actions are modelled as first-order processes:

$$
\begin{aligned}
\frac{\mathrm{d} x_{1}}{\mathrm{~d} t} & =-k_{a 1} x_{1}(t)+k_{b 1} I(t) \\
\frac{\mathrm{d} x_{2}}{\mathrm{~d} t} & =-k_{a 2} x_{2}(t)+k_{b 2} I(t) \\
\frac{\mathrm{d} x_{3}}{\mathrm{~d} t} & =-k_{a 3} x_{3}(t)+k_{b 3} I(t)
\end{aligned}
$$

where $x_{1}$ represents the effects of insulin on glucose distribution/transport, $x_{2}$ represents the effect on glucose disposal and $x_{3}$ the effect on endogenous glucose production; $k_{a i}$, $i=1, \ldots, 3$ are deactivation rate constants and $k_{b i}, i=$ $1, \ldots, 3$ activation rate constants. It will be considered here an alternative parameterization where $\frac{k_{b 1}}{k_{a 1}}=S_{I T}, \frac{k_{b 2}}{k_{a 2}}=$ $S_{I D}$ and $\frac{k_{b 3}}{k_{a 3}}=S_{I E}$.

Finally, glucose metabolism is represented as the twocompartment system [5]

$$
\begin{aligned}
& \begin{aligned}
& \frac{\mathrm{d} Q_{1}(t)}{\mathrm{d} t}=-F_{01}^{c}(t)-x_{1}(t) Q_{1}(t)+k_{12} Q_{2}(t)- \\
&-F_{R}(t)+U_{\mathrm{G}}(t)+\operatorname{EGP}_{0}\left(1-x_{3}(t)\right) \\
& \frac{\mathrm{d} Q_{2}(t)}{\mathrm{d} t}= x_{1}(t) Q_{1}(t)-\left(k_{12}+x_{2}(t)\right) Q_{2}(t) \\
& G(t)=\frac{Q_{1}(t)}{V_{G}}
\end{aligned}
\end{aligned}
$$

where $Q_{1}$ and $Q_{2}$ represent the masses of glucose in the accessible and non-accessible compartments, $k_{12}$ represents the transfer rate constant from the non-accessible to the accessible compartment, $V_{G}$ represent the distribution volume of the accessible compartment, $G$ is the glucose concentration and $\mathrm{EGP}_{0}$ represents endogenous glucose production extrapolated to the zero insulin concentration. $F_{01}^{c}$ is the total non-insulin-dependent glucose disposal, and $F_{R}$ is the

\begin{tabular}{|c|c|c|c|}
\hline Symbol & Quantity & Value & Unit \\
\hline $\bar{A}$ & $\begin{array}{l}\begin{array}{l}\text { Carbohydrate } \\
\text { bioavailability }\end{array} \\
\text { bion) }\end{array}$ & 0.8 & unitless \\
\hline$t_{\max , G}$ & $\begin{array}{l}\text { Time-to-maximum of } \\
\text { CHO absorption }\end{array}$ & 40 & $\min$ \\
\hline$\overline{V_{I}}$ & $\begin{array}{ll}\text { Insulin } & \text { distribution } \\
\text { volum } & \\
\end{array}$ & 0.12 & $\mathrm{~L} / \mathrm{Kg}$ \\
\hline$k_{e}$ & $\begin{array}{l}\text { Insulin elimination } \\
\text { from plasma }\end{array}$ & 0.138 & $1 / \mathrm{min}$ \\
\hline$k_{a 1}$ & Deactivation rate & $0.0157(12)^{*}$ & $1 / \mathrm{min}$ \\
\hline$k_{a 2}$ & Deactivation rate & $0.0231(27)$ & $1 / \mathrm{min}$ \\
\hline$k_{a 3}$ & Deactivation rate & $0.0143(6)$ & $1 / \mathrm{min}$ \\
\hline$\overline{S_{I T}}$ & $\begin{array}{l}\text { Insulin sensitivity of } \\
\text { distribution/transport }\end{array}$ & $\begin{array}{l}18.7 \times 10^{-4} \\
(11)\end{array}$ & $\min ^{-1}$ per $\mathrm{mUL}^{-1}$ \\
\hline$S_{I D}$ & $\begin{array}{l}\text { Insulin sensitivity of } \\
\text { disposal }\end{array}$ & $\begin{array}{l}6.1 \times 10^{-4} \\
(8)\end{array}$ & $\min ^{-1}$ per $\mathrm{mUL}^{-1}$ \\
\hline$\overline{S_{I E}}$ & $\begin{array}{l}\text { Insulin sensitivity of } \\
E G P\end{array}$ & $\begin{array}{l}379 \times 10^{-4} \\
(2)\end{array}$ & $\mathrm{mUL}^{-1}$ \\
\hline$k_{12}$ & Transfer rate & $0.0871(8)$ & $1 / \mathrm{min}$ \\
\hline$f_{01}$ & $\begin{array}{ll}\text { Non-insulin- } \\
\text { dependent } \\
\text { flux }\end{array}$ & $0.0075(2)$ & $\mathrm{mmol} /(\mathrm{kg} \mathrm{min})$ \\
\hline$\overline{V_{G}}$ & $\begin{array}{l}\text { Glucose distribution } \\
\text { volume }\end{array}$ & $0.13(1)$ & $\mathrm{L} / \mathrm{kg}$ \\
\hline $\mathrm{EGP}_{0}$ & $\begin{array}{l}\text { EGP } \quad \text { extrapoled } \\
\text { to zero insulin } \\
\text { concentration }\end{array}$ & $0.0143(2)$ & $\mathrm{mmol} /(\mathrm{kg} \mathrm{min})$ \\
\hline
\end{tabular}
renal glucose clearance above the glucose threshold of 9 mmolL ${ }^{-1}$. Contrary to [5], these ones are modelled by the functions:

$$
\begin{aligned}
& F_{01}^{c}(t)=\frac{f_{01}}{9}\left(G(t)-4.5-\sqrt{(G(t)-4.5)^{2}}\right)+f_{01} \\
& F_{R}(t)=\frac{0.003 V_{G}}{2}\left(G(t)-9+\sqrt{(G(t)-9)^{2}}\right)
\end{aligned}
$$

Table I lists the model parameters used for the different components of the model, taken from [4], [5], patient $n=2$.

TABLE I

PARAMETERS OF THE GLUCORREGULATORY MODEL

*Accuracy of a parameter estimate expressed as a fractional standard deviation (\%).

\section{INTERVAL SIMULATION}

The simulation of a model with particular real values of the parameters, starting from any initial state, yields trajectories across time of the output variables. When the quantities involved in the simulation take values inside intervals of variation, the set of trajectories determine a plane band bounded by two envelopes. At each time step of the simulation, the envelopes, i.e. the possible maximum and minimum values of the variable, have to be determined. This is a range computation problem. The function whose range has to be determined is defined by the interval model of the system and the parameter space is determined by the interval values of the parameters, the input and the initial state. The simulation of an interval model provides intervals (ranges) which can be estimates of the envelopes. A way to compute these estimates is by means of interval arithmetic.

Interval arithmetic [7], [6] considers the whole range of possible instances represented by an interval model. The 
computations of the natural extension of a real function is done by substituting real numbers by intervals and real operations by their interval extensions. An important property is monotonic inclusion: given $f$, a real function, and $f_{R}(X)$ its natural extension to interval $X$, then $x \in X$ implies $f(x) \in f_{R}(X)$.

In consequence, the natural extension is very useful to compute the range of a function because it guarantees on the result. Unfortunately, it does not provide the exact estimate in a general case. This comes from the multi-incidence problem: interval arithmetic considers each instance of a variable in the syntax tree of a function as being independent of each other, leading to an overestimation of the actual range.

In this paper, the interval model is studied using Modal Interval Analysis (MIA) [2], allowing to compute a tight (sometimes exact) enclosure of the envelope that includes all the possible behaviors of the system.

Using modal intervals, each interval function to be evaluated is automatically analyzed and put, if possible, in its optimal form (the expression is rewritten a such a way that the exact range is obtained). Then, the Modal Interval Library IvalDB computes the exact range. In case optimality cannot be reached, the $f^{*}$ algorithm [3] is launched. This algorithm takes benefit of many optimality and coercion theorems from Modal Interval Theory to compute tight approximations of the range by using branch-and-bound techniques.

\section{A. Carbohydrates digestion and absorption}

The glucose absorption model presents uncertainties because the patients generally do not know the exact amount and composition of their meals, which they have to estimate. In addition, the absorption time depends on the patient and the composition of the meal, and therefore it is also an approximation.

It will be thus considered that $D_{G}=\left[\underline{D}_{G}, \bar{D}_{G}\right], A_{G}=$ $\left[\underline{A}_{G}, \bar{A}_{G}\right]$ and $t_{\max , G}=\left[\underline{t}_{\max , G}, \bar{t}_{\max , G}\right]$ in (1). Then, the corresponding absorption interval model is

$$
\begin{aligned}
& {\left[\underline{U}_{\mathrm{G}}(t), \bar{U}_{\mathrm{G}}(t)\right]=} \\
& =\frac{t *\left[\underline{D}_{G}, \bar{D}_{G}\right] *\left[\underline{A}_{G}, \bar{A}_{G}\right] * \exp \left(-t /\left[\underline{t}_{\max , G}, \bar{t}_{\max , G}\right]\right)}{\left[\underline{t}_{\max , G}, \bar{t}_{\max , G}\right]^{2}}
\end{aligned}
$$

where $*$ denotes the interval product and $\exp (\cdot)$ and $(\cdot)^{2}$ are the interval counterparts of the corresponding real functions. As in this equation, $t_{\max , G}$ appears twice (it is multiincident) and (1) do not verify the monotonicity conditions to obtain an optimal computation by means of optimality theorems of MIA, an optimal computation of the range of the glucose absorption rate, $\left[\underline{U}_{\mathrm{G}}(t), \bar{U}_{\mathrm{G}}(t)\right]$, is not possible. Then, the $f^{*}$ algorithm must be used to obtain tight upper and lower envelopes of $U_{G}$.

\section{B. Insulin pharmacokinetics and pharmacodynamics}

The insulin pharmacokinetics model (2) is easy to compute as the only parameter that can be considered as interval is $k_{e}$. In this case, the computations of $I(t)$ are straightforward using IvalDB. Insulin pharmacodynamics (3) is a more complex interval model, as uncertainty is in many parameters.

In case only insulin sensitivity parameters are considered as intervals, $S_{I T}=\left[\underline{S}_{I T}, \bar{S}_{I T}\right], S_{I D}=\left[\underline{S}_{I D}, \bar{S}_{I D}\right]$ and $S_{I E}=\left[\underline{S}_{I E}, \bar{S}_{I E}\right]$, then the discrete interval model

$$
\begin{aligned}
& X_{1}(t+1)=X_{1}(t) *\left(1-t * k_{a 1}\right)+t * S_{I T} * k_{a 1} * I(t) \\
& X_{2}(t+1)=X_{2}(t) *\left(1-t * k_{a 2}\right)+t * S_{I D} * k_{a 2} * I(t) \\
& X_{3}(t+1)=X_{3}(t) *\left(1-t * k_{a 3}\right)+t * S_{I E} * k_{a 3} * I(t)
\end{aligned}
$$

is optimal because the insulin sensibilities appear only once in each equation. As result of the interval computation, the states $X_{1}, X_{2}$, and $X_{3}$ will be interval states.

In case uncertainty is also considered in the deactivation parameters, $K_{a i}=\left[\underline{k}_{a i}, \bar{k}_{a i}\right], i=1, \ldots, 3$, the interval model (3) is not optimal. Upper and lower bound for $X_{i}$ are obtained using the $f^{*}$ algorithm.

\section{Glucose subsystem.}

The output of the carbohydrates digestion and absorption subsystem, $U_{G}$, as well as the computed states from the insulin subsystem, $X_{i}$, will be interval arguments of the glucose subsystem. So, even in the case uncertainty in the parameters of the glucose subsystem is not considered, it becomes an interval model and interval methods should be used to compute its evolution.

Additional parameters of the glucose system that have been considered as intervals are $\mathrm{EGP}_{0}, f_{01}$, and $k_{12}$. In the general case, it is not possible to get exact computations and the $f^{*}$ algorithm should be always used. Exact computations are obtained for $Q_{2}$ using IvalDB, while $f^{*}$ algorithm is needed to compute $Q_{1}$

$$
\begin{aligned}
& \begin{aligned}
Q_{1}(t+1)= & -F_{01}^{c}(t) * t+Q_{1}(t) *\left(1-t * X_{1}(t)\right)+ \\
& +t *\left(k_{12} * Q_{2}(t)-F_{R}(t)+U_{\mathrm{G}}(t)+\right. \\
& \left.+\mathrm{EGP}_{0} *\left(1-X_{3}(t)\right)\right) \\
Q_{2}(t+1)= & Q_{2}(t) *\left(1-t *\left(k_{12}+X_{2}(t)\right)\right)+ \\
& +t * X_{1}(t) * Q_{1}(t)
\end{aligned} \\
& G(t)=\frac{Q_{1}(t)}{V_{G}}
\end{aligned}
$$

\section{Initial states.}

To run the interval simulation of the whole system, initial states and inputs should be given. In this work, three days has been simulated for the given therapy. Starting from a real initial state, it will be considered that at the beginning of the third day the interval values of the states do represent the set of possible initial states the patient may have.

\section{RESULTS}

To show the feasibility of the interval approach, a virtual patient with nominal parameters as described in previous sections has been considered. For this patient, the following typical one day routine has been implemented:

- Food intake: 50 gr CHO at 6:40; 17 gr CHO at 10:00; 137 gr $\mathrm{CHO}$ at 12:20 and 100 gr $\mathrm{CHO}$ at 19:30 
- Basal dosage (Protaphane ${ }^{\circledR}$ ): $8 \mathrm{IU}$ at $6: 20$ and $7 \mathrm{IU}$ at 19:10

- Bolus (Actrapid ${ }^{\complement}$ ): 5IU at 6:20; $9 \mathrm{IU}$ at 12:15 and $6 \mathrm{IU}$ at 19:20

Different scenarios have been considered:

- Scenario 1: $10 \%$ variation in the carbohydrates digestion and absorption model parameters $\left(D_{G}, A_{G}\right.$ and $\left.t_{\max , G}\right)$.

- Scenario 2:

- 2a) $10 \%$ variation in the insulin PK/PD parameters $\left(S_{I D}\right.$ and $\left.S_{I E}\right)$;

- 2b) Scenario 2a with $20 \%$ variation in the insulin PK/PD parameters;

- 2c) Scenario 2a with 8IU instead of 9IU at 12:15.

- Scenario 3: $10 \%$ variation in both, carbohydrates digestion and absorption model parameters and insulin PK/PD parameters.

Figures 2 to 4 show upper and lower bands of the plasma gluc

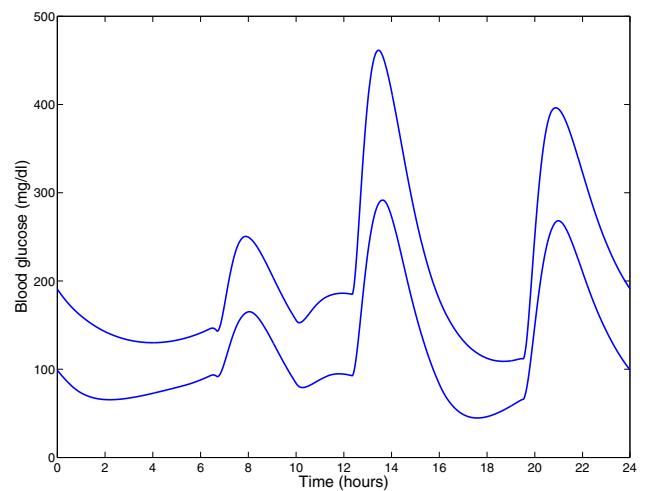

Fig. 2. Envelopes obtained for $10 \%$ variation in $D_{G}, A_{G}$ and $t_{\max , G}$.

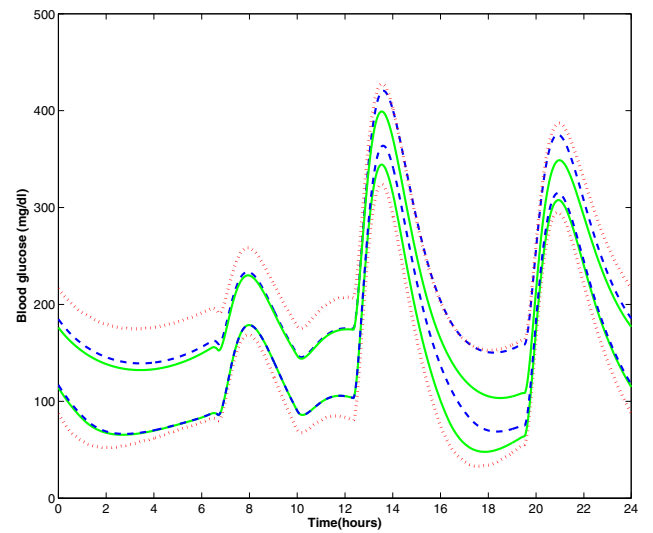

Fig. 3. Envelopes obtained for Scenarios 2a (solid line); $2 \mathrm{~b}$ (dotted line) and $2 \mathrm{c}$ (dashed line).

As it may be observed, consideration of uncertainty reveals the chance of suffering a severe hypoglycemia around 5 p.m. as well as two hyperglycaemias around 2 p.m. and 9 p.m. Hypoglycaemia risk can be reduced significantly by decreasing in 1 IU the bolus dose at 12:15 without affecting too much the hyperglycaemias.

\section{CONCLUSIONS AND FUTURE WORKS}

Modal interval analysis has successfully been applied to the prediction of glucose excursions in patients with type 1

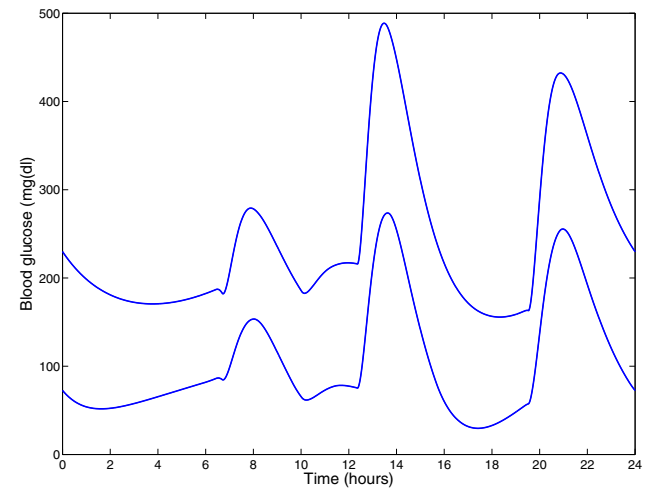

Fig. 4. Envelopes obtained for $10 \%$ variation in $D_{G}, A_{G}, t_{\max , G}, S_{I D}$ and $S_{I E}$.

diabetes face to uncertain information. Uncertain parameters such as hepatic and peripheral insulin sensitivities as well as food intake have been considered for the prediction of the effects of a given insulin therapy on glycaemia. As result, a band containing all possible glucose excursions suffered by the patient for the given uncertainty is obtained.

By considering intra-patient variability and uncertainty in the food intake, a safer prediction of possible hyperand hypoglycaemia episodes induced by the tested insulin therapy can be calculated, leading to a reduction in the number of false-negatives.

Currently, the methods here developed are being integrated in a model-based decision-aid system for the suggestion of bolus insulin doses and injection time which minimize the risk of postprandial hyper- and hypoglycaemia in patients with type 1 diabetes.

\section{REFERENCES}

[1] The Diabetes Control and Complications Trial Research Group, The effect of intensive treatment of diabetes on the development and progression of long term complications in insulin-dependent diabetes mellitus, New England Journal of Medicine, vol. 329, n. 14, pp. $977-$ 986, 1993.

[2] Gardeñes, E. and Sainz, M.A. and Jorba, L. and Calm, R. and Estela, R. and Mielgo, H. and Trepat, A., Modal Intervals, Reliable Computing, vol. 7, n. 2, pp. 77-111, 2001.

[3] Herrero, P. and Sainz, M.A. and Vehi, J. and Jaulin, L., Quantified Set Inversion Algorithm with Applications to Control, Reliable Computing, vol. 11, n. 5, pp. 369-382, 2005.

[4] Hovorka, R. and Shojaee-Moradie, F. and Carroll, PV. and Chassin, LJ. and Gowrie, IJ. and Jackson, NC. and Tudor, RS. and Umpleby, AM. and Jones, RH., Partitioning glucose distribution/transport, disposal, and endogenous production during IVGTT, American Journal of Physiology, Endocrinology and Metabolism, vol. 282, n. 5, pp. 9921007, 2002.

[5] Hovorka, R. and Canonico, V. and Chassin, LJ. and Haueter, U. and Massi-Benedetti, M. and Orsini Federici, M. and Pieber, TR. and Schaller, HC. and Schaupp, L. and Vering, T. and Wilinska, ME., Nonlinear model predictive control of glucose concentration in subjects with type 1 diabetes, Physiological Measurement, vol. 24, n. 4, pp. 905-920, 2004

[6] Jaulin, L., Kieffer, M., Didrit O., and Walter E. (2001). Applied Interval Analysis. Springer, London.

[7] Moore, Ramon E, "Interval analysis", Prentice-Hall Inc., 1966.

[8] Tarín, C. and Teufel, E. and Picó, J. and Bondia, J. and Pfleiderer, J., "Comprehensive Pharmacokinetic Model of Insulin Glargine and Other Insulin Formulations", IEEE Transactions on Biomedical Engineering, vol. 52, n. 12, pp. 1994-2005, 2005. 